\title{
BMJ Open Resuming anticoagulants after anticoagulation-associated intracranial haemorrhage: systematic review and meta-analysis
}

\author{
Zien Zhou, ${ }^{1,2}$ Jie Yu, ${ }^{2,3}$ Cheryl Carcel, ${ }^{2,4,5}$ Candice Delcourt, ${ }^{2,4,5}$ Jiehui Shan, ${ }^{6}$ \\ Richard I Lindley, ${ }^{2,5}$ Bruce Neal, ${ }^{2,7,8}$ Craig S Anderson, ${ }^{2,4,9}$ Maree L Hackett ${ }^{2,5}$
}

To cite: Zhou Z, Yu J, Carcel C, et al. Resuming anticoagulants after anticoagulation-associated intracranial haemorrhage: systematic review and meta-analysis. BMJ Open 2018;8:e019672. doi:10.1136/ bmjopen-2017-019672

- Prepublication history and additional material for this paper are available online. To view these files, please visit the journal online (http://dx.doi. org/10.1136/bmjopen-2017019672).

ZZ and JY contributed equally.

Received 22 September 2017 Revised 27 March 2018 Accepted 10 April 2018

Check for updates

For numbered affiliations see end of article.

Correspondence to

Dr Zien Zhou;

zzhou@georgeinstitute.org.au

\section{ABSTRACT}

Objective To determine the adverse outcomes following resumption of anticoagulation in patients with anticoagulation-associated intracranial haemorrhage (ICH).

Design We performed a systematic review and metaanalysis in this clinical population. The Preferred Reporting Items for Systemic Reviews and Meta-Analyses statement was followed, and two authors independently assessed eligibility of all retrieved studies and extracted data.

Data sources Medline, Embase and the Cochrane Central Register of Controlled Trials, from inception to February 2017.

Eligibility criteria and outcomes Randomised controlled trials or cohort studies that recruited adults who received oral anticoagulants at the time of ICH occurrence and survived after the acute phase or hospitalisation were searched. Primary outcomes, including long-term mortality, recurrent ICH and thromboembolic events. Secondary outcomes were the frequency of resuming anticoagulant therapy and related factors.

Results We included 12 cohort studies (no clinical trials) involving 3431 ICH participants. The pooled frequency of resuming anticoagulant therapy was $38 \%$ (95\% $\mathrm{Cl} 32 \%$ to $44 \%$ ), but this was higher in participants with prosthetic heart valves, subarachnoid haemorrhage or dyslipidaemia. There was no evidence that resuming anticoagulant therapy was associated with higher long-term mortality (pooled relative risk (RR) $0.60,95 \% \mathrm{Cl} 0.30$ to 1.19 ; $\mathrm{p}=0.14$ ) or ICH recurrence (pooled RR $1.14,95 \% \mathrm{Cl} 0.72$ to $1.80 ; p=0.57$ ). Resumption of anticoagulation was associated with significantly fewer thromboembolic events (pooled RR $0.31,95 \% \mathrm{Cl} 0.23$ to $0.42 ; \mathrm{p}<0.001$ ). In a subgroup of patients with atrial fibrillation, resuming anticoagulant therapy was associated with fewer longterm mortality (pooled RR $0.27,95 \% \mathrm{Cl} 0.20$ to 0.37 , $\mathrm{p}<0.001$ ).

Conclusions Based on these observational studies, resuming anticoagulant therapy after anticoagulationassociated ICH has beneficial effects on long-term complications. Clinical trials are needed to substantiate these findings.

PROSPERO registration number CRD42017063827.
Strengths and limitations of this study

- A greater number of potentially eligible articles were screened and included for pooling.

- We paid careful attention to include participants who received anticoagulant therapy at the time of intracranial haemorrhage occurrence and survived the acute phase or hospitalisation.

- There are many confounders (anticoagulation indication, age, clinical severity, haematoma location, etc) related to resumption of anticoagulation in existing observational studies.

- The confounders cannot be adjusted in this study-level meta-analysis which limits the formation of management recommendations or improve clinical practice.

\section{INTRODUCTION}

Oral anticoagulant is recommended for patients with atrial fibrillation (AF) or prosthetic heart valves (PHV) to prevent cardioembolic stroke or systemic embolism. Severe bleeding is the major fatal complication of anticoagulant therapy, occurring in $0.3 \%-$ $4.5 \%$ per year for gastrointestinal (GI) tract bleeding ${ }^{12}$ and $0.3 \%-0.7 \%$ per year for intracranial haemorrhage (ICH). ${ }^{34}$ Although GI bleeding is more common than ICH as a complication of anticoagulant therapy, the main cause of bleeding-related morbidity and mortality is ICH. ${ }^{56}$ Furthermore, anticoagulation-associated ICH is associated with worse prognosis than spontaneous ICH. ${ }^{78}$

Once severe bleeding occurs and anticoagulant therapy is interrupted, whether and when to resume anticoagulant therapy is a common therapeutic dilemma, since these patients remain at high thromboembolic risk. A meta-analysis showed that resumption of warfarin following interruption because of anticoagulation-associated GI bleeding is associated with a reduction in thromboembolic 
events and mortality without a statistically significant increase in recurrent GI bleeding. ${ }^{9}$ However, evidence for resumption of anticoagulant therapy after anticoagulation-associated ICH is not sufficiently reliable to guide clinical practice, especially as the evidence was partly based on observational case series, ${ }^{10-12}$ rather than randomised controlled trials (RCTs). In this systematic review and meta-analysis, we aim to provide an updated overview on the risks of long-term mortality, recurrent haemorrhage and thromboembolic complications following resumption of anticoagulation in patients who experience anticoagulation-associated ICH and survive the acute phase or hospitalisation.

\section{METHODS}

\section{Patient and public involvement}

Patients and public were not involved in this study.

\section{Search strategy}

The Preferred Reporting Items for Systemic Reviews and Meta-Analyses statement ${ }^{13}$ was followed. The protocol was registered with the International Prospective Register of Systematic reviews (CRD42017063827). We searched Medline, Embase and the Cochrane Central Register of Controlled Trials from inception to February 2017. Our search strategy was based on the combination of the following medical subject headings terms or keywords: ['anticoagulant' OR 'platelet aggregation inhibitors' OR 'atrial fibrillation' OR 'heart valve prosthesis' OR 'venous thromboembolism' OR 'pulmonary embolism'] AND ['intracranial haemorrhage' OR 'cerebral hemorrhage'] AND ['resum*' OR 'recur*' OR 'reinitiat*' OR 're-initiat*' OR 'interupt*'] (online supplementary method S1). The ClinicalTrials.gov website was also searched for randomised trials that were registered as completed but not yet published. There was no language restriction. Reference lists of all retrieved studies and related review articles were cross-checked for further relevant studies until no further publications were found.

\section{Eligibility criteria}

We included RCTs or cohort studies that recruited participants ( $\geq 18$ years) who received oral anticoagulants at the time of ICH occurrence and survived after the acute phase or hospitalisation. Oral anticoagulants included vitamin $\mathrm{K}$ antagonist (VKA) and non-vitamin $\mathrm{K}$ antagonist (NOAC). ICH was identified through appropriate brain imaging documentation. Data on thromboembolic events, recurrent ICH or long-term mortality after resuming anticoagulant therapy can be extracted from the reports. We excluded the following studies: (1) where outcomes were not reported separately for participants who did and did not resume anticoagulant therapy (or switched to antiplatelet agents); (2) included mixed populations (such as with ICH and GI bleeding) and separate results for the participants with ICH could not be identified; (3) the primary aim was to assess risks of resuming anticoagulation following traumatic ICH or surgical intervention for ICH and (4) reviews, editorials, letters, case or case series reports, guidelines, technical notes and book chapters.

\section{Study selection and data extraction}

The review of potentially eligible RCTs or cohort studies identified by the searches was conducted by two authors (ZZ and JY) to identify reports for review in full text. Each full-text article was then reviewed for eligibility by these authors and, for each included study, data were extracted independently and in duplicate using a standardised electronic form. Any disagreement on extracted data was settled by discussion or in consultation with two other authors (JS and MLH). Extracted data included: (1) first author, year of publication, country or region, study design, sample size and clinical characteristics of recruited participants at baseline (age, gender, hypertension, diabetes mellitus, dyslipidaemia, coronary artery disease, heart failure, history of stroke and current smoking status); (2) anticoagulation indication, type of anticoagulant and whether antiplatelet agents are coprescribed; (3) diagnostic method of ICH, location of ICH, number of participants with and without resuming anticoagulant therapy after ICH, and duration of anticoagulant interruption after ICH and (4) length of follow-up, number of lost to follow-up, long-term mortality, recurrent ICH and thromboembolic events in patients who did and did not resume anticoagulant therapy. Two authors (ZZ and JY) also judged the quality of each included RCT or cohort study according to the Cochrane Collaboration's tool ${ }^{14}$ or the Newcastle-Ottawa Scale $(\mathrm{NOS})^{15}$ for assessing risk of bias.

\section{Outcomes}

The primary outcomes of interest (for RCTs and cohort studies) were long-term mortality, recurrent ICH and thromboembolic events (such as deep vein thrombosis (DVT), pulmonary embolism (PE), ischaemic stroke (IS), myocardial infarction (MI) or systemic embolisation) after the acute phase or hospitalisation. The secondary outcomes (for cohort studies) were: (1) frequency of resumption of anticoagulant therapy after anticoagulation-associated ICH, and (2) factors (demographics, comorbidities, anticoagulation indication, coprescribing of antiplatelet agents and location of ICH at baseline) associated with resuming anticoagulant therapy after anticoagulation-associated ICH.

\section{Statistical analysis}

The number of dichotomous outcomes were summarised and mean values with SDs were collated for continuous outcomes. Pooled relative risks (RRs) with 95\% CIs were estimated for primary and secondary outcomes using the DerSimonian and Laird random-effects model. ${ }^{16}$ In every case, a two-sided $p$ value of $\leq 0.05$ was deemed significant. The percentage of variability across the pooled estimates attributable to heterogeneity beyond chance 
was estimated using the $\mathrm{I}^{2}$ statistic and by calculating the $\mathrm{p}$ value for heterogeneity. $\mathrm{I}^{2}$ values of $25 \%, 50 \%$ and $75 \%$ were regarded as low, moderate and high heterogeneity, respectively. Where there was a high likelihood of differences, sensitivity analyses or subgroup analyses were performed by excluding individual studies or stratification of potential confounding factors (study design, anticoagulation indication, ICH location, etc) to determine reasons for the differences. Random-effects meta-regression on primary outcomes with potential heterogeneity were conducted to investigate the association between the observed RR and duration of anticoagulation interruption or follow-up length for each study. In addition, we made three sets of comparisons on primary outcomes among participants: (1) who resumed anticoagulant therapy versus did not resume, (2) who resumed anticoagulant therapy versus switched to antiplatelet agents and (3) who switched to antiplatelet agents versus did not resume anticoagulant therapy. Evidence of publication bias was sought using Egger's regression test for funnel asymmetry in addition to visual inspection of the funnel plots. All statistical analyses were performed using Stata V.12.0.

\section{RESULTS}

\section{Study selection and characteristics}

The literature search yielded 5183 potentially eligible articles or conference abstracts, of which 47 articles were reviewed in full text (figure 1). Of these, 12 studies (no RCTs, 12 cohorts) ${ }^{17-28}$ including 3431 participants (1141 resumed anticoagulant therapy, 1500 did not resume anticoagulant therapy, 790 switched to antiplatelet agents) met the inclusion criteria. During follow-up, a total of 598 participants died after the acute phase or hospitalisation. There were 207 events of ICH recurrence and 308 thromboembolic events reported. Most excluded studies were not original research, were survey or decision analysis

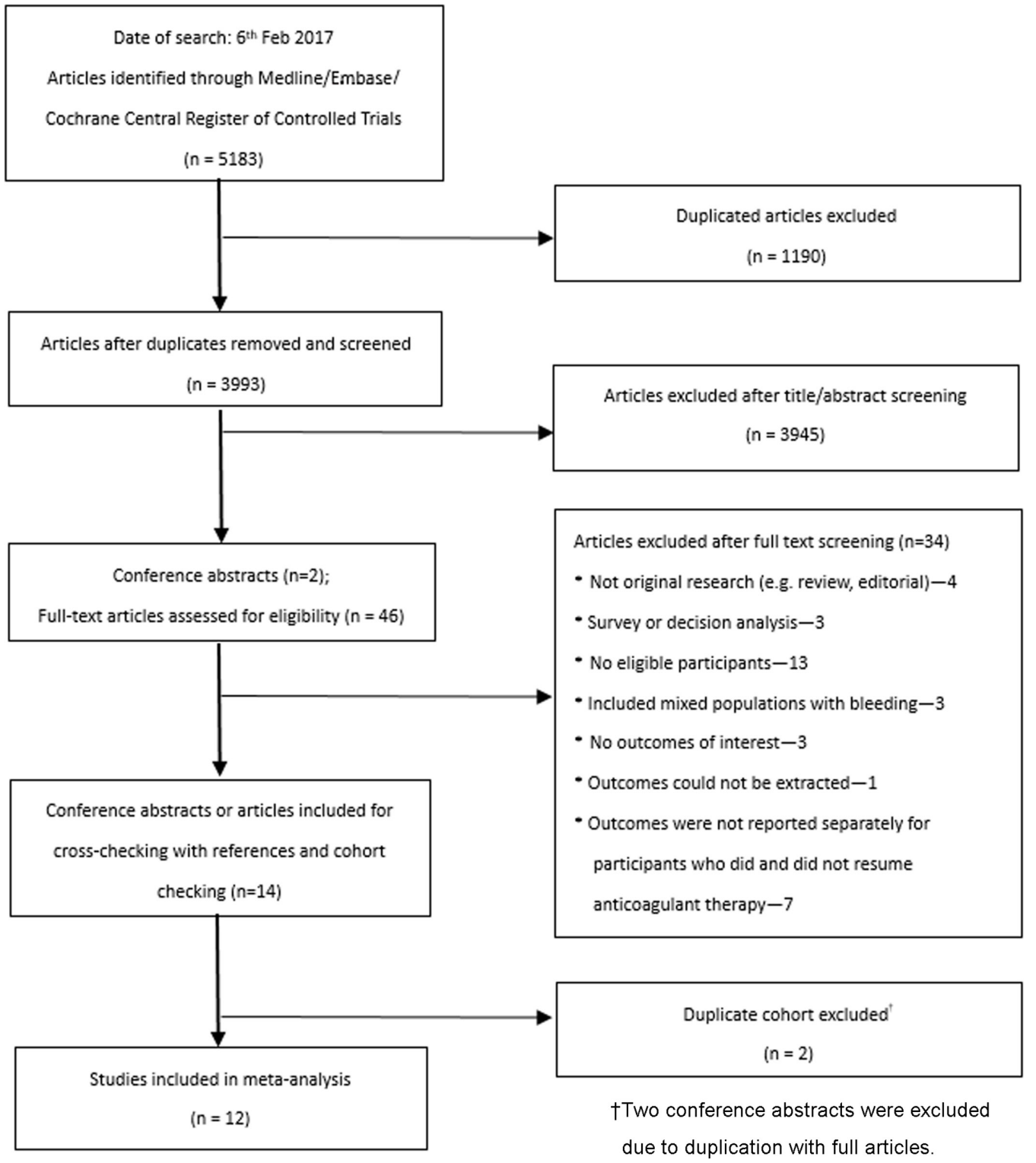

Figure 1 Flow chart of literature search. 


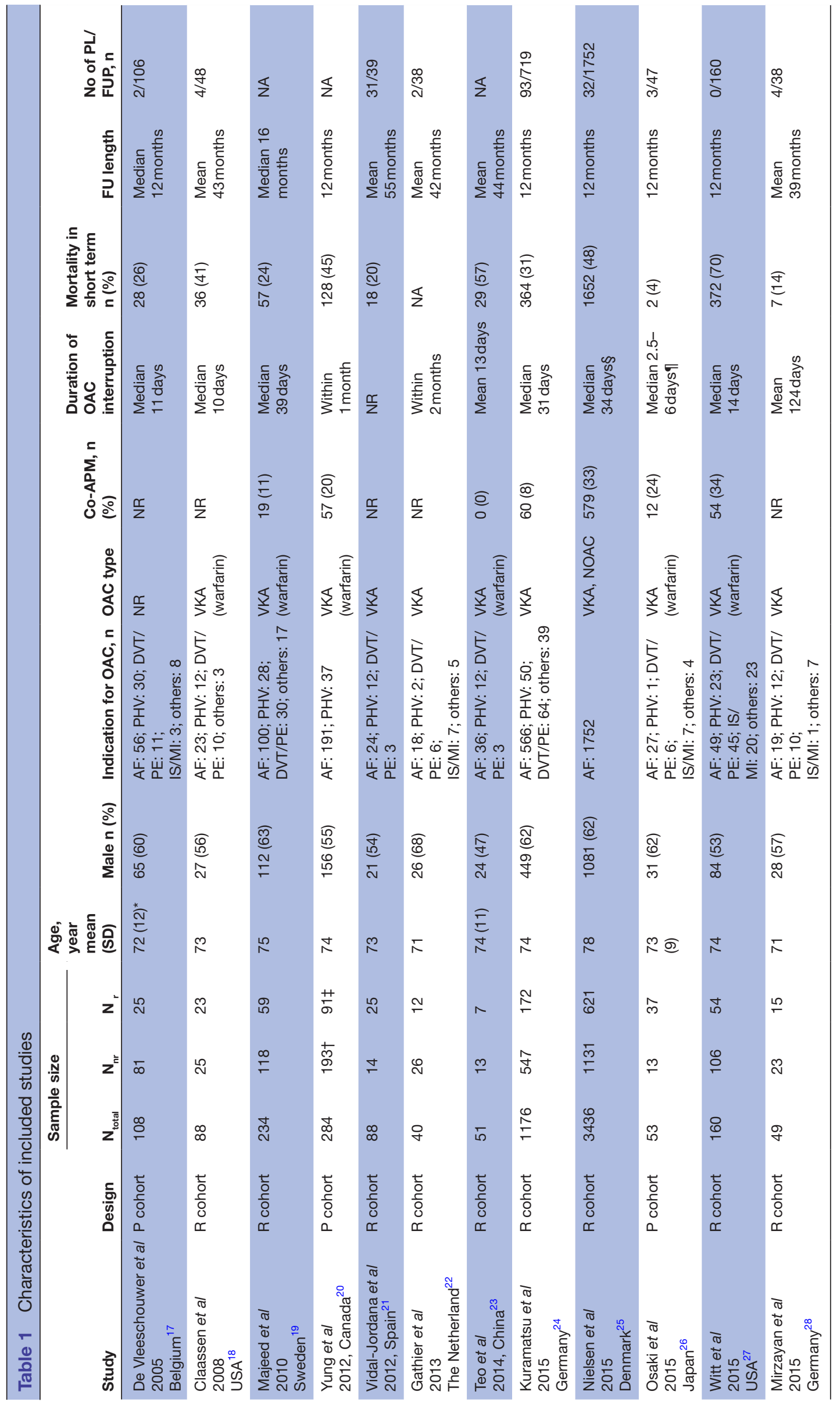

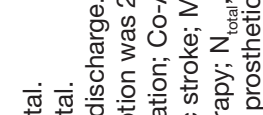

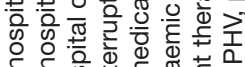

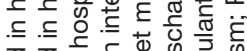

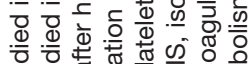

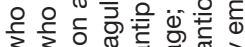

की की 응.

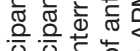

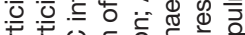

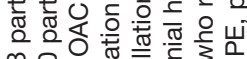

त象

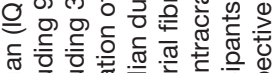

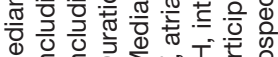

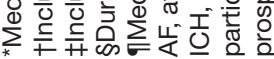


papers, without eligible participants, included mixed populations with bleeding or without eligible outcomes of interest (online supplementary table S1). Two conference abstracts were excluded due to duplication with full articles.

Included studies were published from 2005 to 2016, and sample sizes ranged from $20^{23}$ to 1752 participants, ${ }^{25}$ with the length of follow-up ranging from $12^{172024-27}$ to 55 months $^{21}$ (table 1, online supplementary table S2). The type of oral anticoagulants received at the time of ICH occurrence was VKA in all studies, with the exception of De Vleeschouwer et al ${ }^{17}$ (the type of anticoagulation was not reported) and Nielsen et $a 2^{25}$ (some participants received NOAC). The NOS quality assessment characteristics are shown in (online supplementary table S3), with many studies demonstrating a high degree of selection towards those resuming anticoagulation.

Associations of resuming anticoagulant therapy with primary outcomes

Mean or median duration of anticoagulation interruption in participants who resumed anticoagulant therapy ranged between $2.5^{26}$ and 124 days $^{28}$ after ICH. Three studies did not report the exact timing of resuming anticoagulant therapy. ${ }^{20-22}$ Seven studies ${ }^{102022425} 27$ could be pooled for long-term mortality and 11 studies $^{17-19}{ }^{21-28}$ were pooled for other primary outcomes. Compared with resuming anticoagulant therapy or switching to antiplatelet agents after ICH, there was no significant association of resuming anticoagulant therapy with higher risks
Source

Long-term mortality

Claassen, 2008 [18]

Yung, 2012 [20]

Vidal-Jordana, 2012 [21]

Gathier, 2013 [22]

Kuramatsu, 2015 [24]

Nielsen, 2015 [25]

Witt, 2015 [27]

Total $\quad(1-$ squared $=86.5 \%, p=0.000)$

\section{$\mathrm{ICH}$ recurrence}

De Vleeschouwer, 2005 [17]

Claassen, 2008 [18]

Majeed, 2010 [19]

Vidal-Jordana, 2012 [21]

Gathier, 2013 [22]

Teo, 2014 [23]

Kuramatsu, 2015 [24]

Nielsen, 2015 [25]

Osaki, 2015 [26]

Witt, 2015 [27]

Mirzayan, 2016 [28]

Total $\quad(I-$ squared $=29.3 \%, p=0.166)$

TEE complications

De Vleeschouwer, 2005 [17]

Claassen, 2008 [18]

Majeed, 2010 [19]

Vidal-Jordana, 2012 [21]

Gathier, 2013 [22]

Teo, 2014 [23]

Kuramatsu, 2015 [24]

Nielsen, 2015 [25]

Osaki, 2015 [26]

Witt, 2015 [27]

Mirzayan, 2016 [28]

Total $(\mathrm{I}$-squared $=0.0 \%, p=0.838)$
Favours re-AC

Favours not re-AC

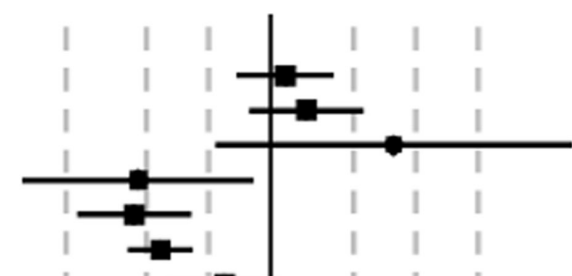

$\mathrm{RR}(95 \% \mathrm{Cl})$

$\%$ Weight

Figure 2 Meta-analysis of primary outcomes. $\mathrm{ICH}$, intracranial haemorrhage; re-AC, resumption of anticoagulant therapy; RR, relative risk; TEE, thromboembolic events. 


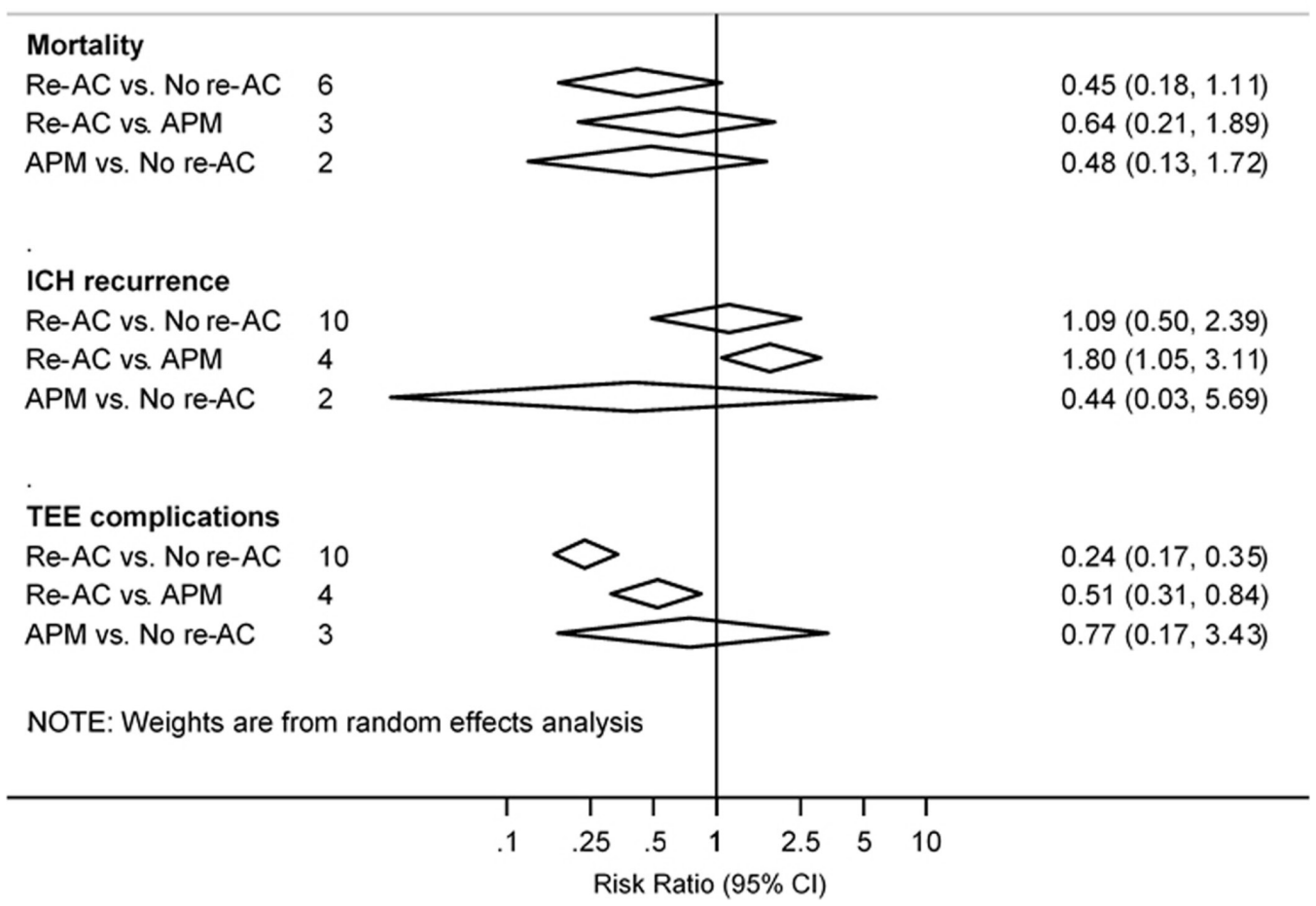

Figure 3 Analysis on primary outcomes among participants who switched to antiplatelet medication, who did and did not resume anticoagulant therapy after anticoagulation-associated ICH. APM, antiplatelet medication; ICH, intracranial haemorrhage; Re-AC, resumption of anticoagulant therapy; RR, relative risk; TEE, thromboembolic events.

of long-term mortality (pooled RR $0.60,95 \% \mathrm{CI} 0.30$ to $1.19 ; \mathrm{p}=0.14$ ) or ICH recurrence (pooled RR $1.14,95 \%$ CI 0.72 to $1.80 ; \mathrm{p}=0.57$ ) (figure 2 and online supplementary table S4). There were significant associations of resuming anticoagulant therapy with lower risks of thromboembolic events (pooled RR 0.31, 95\% CI 0.23 to $0.42 ; \mathrm{p}<0.001$ ). Compared with switching to antiplatelet agents, resuming anticoagulant therapy was significantly more likely to lead to ICH recurrence (pooled RR 1.80, 95\% CI 1.05 to 3.11; $\mathrm{p}=0.03$ ) (figure 3), but thromboembolic events were significantly more likely to be avoided (pooled RR 0.51 , $95 \%$ CI 0.31 to $0.84 ; \mathrm{p}=0.01$ ). No difference was found between switching to antiplatelet agents and anticoagulation resumption on long-term mortality (pooled RR 0.64, 95\% CI 0.21 to $1.89 ; \mathrm{p}=0.41$ ). Funnel plots and Egger's regression tests identified no strong evidence of publication bias for all primary outcomes except ICH recurrence ( $\mathrm{p}=0.04)$ (online supplementary figure $\mathrm{S} 1$ ).

There was high heterogeneity in results pooled for long-term mortality $\left(I^{2}=86.5 \%\right)$, which remained high in sensitivity analyses that serially excluded each study $\left(\mathrm{I}^{2}\right.$ from $83.6 \%$ to $88.7 \%$, online supplementary table S5), but subgroup analysis including two studies ${ }^{24} 25$ with the anticoagulation indication of AF showed significant benefits without heterogeneity (pooled RR $0.27,95 \%$ CI 0.20 to $0.37 ; \mathrm{p}<0.001 ; \mathrm{I}^{2}=0.0 \%$ ) (online supplementary figure S2). There was potential heterogeneity in results pooled for ICH recurrence $\left(\mathrm{I}^{2}=29.3 \%\right)$. A sensitivity analysis was performed after dropping studies recruiting participants with subarachnoid haemorrhage $(\mathrm{SAH})$ or subdural haemorrhage (pooled RR 1.93, 95\% CI 0.93 to $2.60 ; \mathrm{p}=0.10 ; \mathrm{I}^{2}=0.0 \%$ ) (online supplementary figure S3). Meta-regression identified no association of the RR of long-term mortality with duration of anticoagulation interruption after ICH $(p=0.21)$ or length of follow-up $(\mathrm{p}=0.35)$. Meta-regression also identified no association of the RR of ICH recurrence with duration of anticoagulation interruption $(p=0.41)$ but potential association with length of follow-up ( $\mathrm{p}=0.05$ ) (online supplementary figures S4 and S5).

\section{Meta-analysis of secondary outcomes}

The pooled frequency of resuming anticoagulant therapy after anticoagulation-associated ICH was $38 \%$ (95\% CI 32\% to $44 \%$ ), but there was high heterogeneity $\left(\mathrm{I}^{2}=89.9 \%\right)$ (online supplementary figure $\left.\mathrm{S} 6\right)$. Indications for anticoagulant therapy before $\mathrm{ICH}$ recurrence were $\mathrm{AF}$ ( $31 \%$ to $100 \%$ ), PHV (2\% to $31 \%$ ), DVT or PE (DVT/ PE) $(6 \%$ to $21 \%)$ and IS or MI (IS/MI) (2\% to $18 \%)$ (online supplementary table S6). Participants with PHV were 2.52 (95\% CI 1.83 to 3.45 ) and 2.41 (95\% CI 1.90 to 3.04) times more likely to resume anticoagulant therapy than those with $\mathrm{AF}$ and DVT/PE (table 2 and online supplementary table S6). Participants with DVT/PE were 1.28 (95\% CI 1.07 to 1.52 ) times more likely to resume anticoagulant therapy than those with AF. There was no association between resuming anticoagulant therapy and location of ICH, except resumption was $1.65(95 \%$ CI 1.00 to 2.72) times more likely to occur in participants with SAH than with intracerebral haemorrhage (table 2 
Table 2 Meta-analysis of factors associated with resuming anticoagulant therapy after anticoagulation-associated ICH

\begin{tabular}{|c|c|c|c|c|}
\hline Factors & No of studies & $\begin{array}{l}\text { Included studies } \\
\text { (reference no) }\end{array}$ & RR $(95 \% \mathrm{Cl})$ & Heterogeneity $I^{2}(\%)$ \\
\hline \multicolumn{5}{|l|}{ Anticoagulation indication } \\
\hline $\mathrm{PHV}$ versus $\mathrm{AF}$ & 8 & $18-22242627$ & 2.52 (1.83 to 3.45$)$ & 71.9 \\
\hline DVT/PE versus AF & 7 & 18192122242627 & 1.28 (1.07 to 1.52$)$ & 0.0 \\
\hline IS/MI versus $A F$ & 3 & 222627 & 0.87 (0.56 to 1.36$)$ & 0.0 \\
\hline PHV versus DVT/PE & 7 & 18192122242627 & 2.41 (1.90 to 3.04$)$ & 26.6 \\
\hline IS/MI versus PHV & 3 & 222627 & 0.33 (0.08 to 1.37$)$ & 74.7 \\
\hline IS/MI versus DVT/PE & 3 & 222627 & $0.69(0.32$ to 1.52$)$ & 38.2 \\
\hline \multicolumn{5}{|l|}{ Location of ICH } \\
\hline Lobar versus deep & 6 & $1820-222427$ & 1.04 (0.85 to 1.28$)$ & 0.0 \\
\hline Lobar versus pons/cerebellar & 4 & 18202224 & 1.01 (0.76 to 1.35$)$ & 0.0 \\
\hline Lobar versus intraventricular & 5 & 1820222427 & 1.06 (0.84 to 1.35$)$ & 0.0 \\
\hline Deep versus pons/cerebellar & 4 & 18202224 & 0.96 (0.71 to 1.30$)$ & 0.0 \\
\hline Deep versus intraventricular & 5 & 1820222427 & 1.07 (0.84 to 1.36$)$ & 0.0 \\
\hline Subarachnoid versus $\mathrm{ICH}^{*}$ & 2 & 2027 & 1.65 (1.00 to 2.72$)$ & 49.0 \\
\hline Subarachnoid versus subdural & 2 & 1927 & $1.20(0.80$ to 1.80$)$ & 0.0 \\
\hline \multicolumn{5}{|c|}{ Demographics, comorbidities and baseline characteristics } \\
\hline Male versus female & 6 & $1820-222427$ & $1.08(0.88$ to 1.32$)$ & 20.9 \\
\hline With $\mathrm{HT}$ versus without & 6 & $1820-222427$ & 1.04 (0.85 to 1.28$)$ & 0.0 \\
\hline With DM versus without & 6 & $1820-222427$ & $0.90(0.74$ to 1.10$)$ & 0.0 \\
\hline With DL versus without & 3 & 202124 & $1.23(1.02$ to 1.49$)$ & 0.0 \\
\hline With CAD versus without & 4 & 18202224 & $1.06(0.86$ to 1.30$)$ & 1.0 \\
\hline With HF versus without & 3 & 202427 & $1.03(0.77$ to 1.37$)$ & 0.0 \\
\hline With $\mathrm{Str} / \mathrm{TI} \mathrm{A}^{*}$ versus without & 6 & $18-2224$ & $0.98(0.80$ to 1.21$)$ & 15.4 \\
\hline INR $>3$ versus $I N R<3$ & 2 & 2027 & $1.69(0.44$ to 6.52$)$ & 96.1 \\
\hline With surgery versus without & 2 & 2427 & 0.93 (0.71 to 1.23$)$ & 0.0 \\
\hline With co-APM versus without & 3 & 202427 & 0.90 (0.56 to 1.45$)$ & 64.0 \\
\hline
\end{tabular}

*With history of stroke or TIA.

$\mathrm{AF}$, atrial fibrillation; CAD, coronary artery disease; co-APM, antiplatelet medicine being coprescribed at the time of ICH; DL, dyslipidaemia; DM, diabetes mellitus; DVT/PE: deep vein thrombosis or pulmonary embolism; HF, heart failure; HT, hypertension; ICH, intracranial haemorrhage; $\mathrm{ICH}^{*}$, intracerebral haemorrhage (including haemorrhage at lobar, deep, pons, cerebellar or intraventricular region); INR, international normalised ratio; IS/MI, ischaemic stroke or myocardial infarction; PHV, prosthetic heart valve; re-AC, resumption of anticoagulant therapy; RR, relative risk; Str/TIA, stroke or transient ischaemic attack.

and online supplementary table S7). In consideration of the demographics and comorbidities, participants with dyslipidaemia were $1.23(95 \%$ CI 1.02 to 1.49$)$ times more likely to resume anticoagulant therapy (table 2 and online supplementary table S8).

\section{DISCUSSION}

This systematic review and meta-analysis showed that resuming anticoagulant therapy after interruption for anticoagulation-associated ICH reduced the risks of thromboembolic events, without significantly increasing the risks of ICH recurrence and long-term mortality. For patients with $\mathrm{AF}$, benefit was found on long-term mortality after resuming anticoagulation, which is consistent with the result of a study based on meta-analysis of individual patient data. ${ }^{29}$

Strengths of our study over another three meta-analysis published recently on this topic, ${ }^{30-32}$ included the greater number of potentially eligible articles screened and the subsequent inclusion of more studies for meta-analysis. In addition, we tried to avoid confounding effects of management during the acute phase by focusing on participants who had survived the acute phase or hospitalisation, as long-term outcome is a key factor influencing clinical decision-making. We also focused on studies where patients had received anticoagulant therapy at the time of ICH occurrence and explored the factors related to resume anticoagulant therapy after anticoagulation-associated ICH. One study, ${ }^{33}$ included in the review 
of Murthy et $a l,{ }^{30}$ was excluded from our review because recruited participants were those with specific indications for anticoagulant therapy rather than just receiving the therapy. The results in our review are similar to three others with study-level meta-analysis. ${ }^{30-32}$ A difference of the effect on mortality between our study (non-significant decrease) and that of Chai-Adisaksopha $e t a l$ 's (significant decrease $)^{32}$ might be due to different designs or analysis methods (eg, switching to antiplatelet agents is considered as the comparator in our study but not in Chai-Adisaksopha et al's) (online supplementary table S9).

No RCT was found in our search and only one (APACHE-AF) $^{34}$ is currently in progress, although others are planned (C Anderson, personal communication). Since the evidence is based on observational studies, participant-specific factors (such as anticoagulation indication, ICH characteristics, comorbidities, etc) will lead to selection bias in the decision to resume anticoagulant therapy and confounding effects on clinical outcomes cannot be avoided. We found that anticoagulant therapy was more likely to be represcribed in participants with $\mathrm{PHV}$ and SAH. The decision to resume anticoagulant therapy did not appear to be influenced by ICH location (lobar or deep), although one prior study concluded that patients with deep ICH would be more qualified for resumption of anticoagulation and those with lobar ICH had a higher risk of recurrent ICH after resumption of anticoagulation. ${ }^{35}$ Interestingly, dyslipidaemia was the only comorbidity that was associated with resuming anticoagulation.

The optimal timing to resume anticoagulant therapy after anticoagulation-associated ICH is still uncertain. The results from our meta-regression analyses of duration of anticoagulant interruption on RR of long-term mortality or recurrent ICH should be treated with caution, since the exact duration of anticoagulant interruption after ICH was not reported in some studies and an approximate time was used instead. In addition, we could not adjust for confounding by indication. Majeed $e t a l^{19}$ reported that the optimal timing for resuming anticoagulant therapy was between 10 and 30 weeks after anticoagulation-associated ICH in a study of 177 ICH survivors with different anticoagulation indications. A recent observational study from Sweden reported that the optimal timing was 7-8 weeks after ICH in 2619 ICH survivors with $\mathrm{AF}^{36}$ and current guidelines from the American Heart Association/American Stroke Association suggest 4 weeks is a reasonable delay before resuming anticoagulant therapy (excluding those with PHV). ${ }^{37}$ Evidence for patients with PHV is limited but one survey of 504 physicians showed a preference for resumption between 4 and 14 days after $\mathrm{ICH},{ }^{38}$ while another review based on case reports or case series reported a non-significant trend towards lower ICH recurrence and delayed resumption of anticoagulation after ICH. ${ }^{39}$ Further research stratified by anticoagulation indication and other confounding factors should advance knowledge.

Switching to antiplatelet agents is a popular alternative strategy for patients with anticoagulation-associated ICH.
We note that this produced a benefit in terms of ICH recurrence but was inferior with regard to preventing thromboembolic complications compared with resuming anticoagulant therapy. Two RCTs (RESTART ${ }^{40}$ and RESTART-Fr ${ }^{41}$ ) addressing this question are now being undertaken. We also note that VKA was used in most studies $(10 / 12)$ included in this review, but clinical practice is rapidly changing away from these agents as NOACs become more widely available and familiar. Although evidence indicates that NOACs are safer than VKA in patients with $\mathrm{AF}^{42}{ }^{43}$ unfavourable results are reported in patients with PHV. ${ }^{44} 45$ Patients with NOAC-associated ICH may have better functional outcomes than those with VKA-associated ICH. ${ }^{46}{ }^{47}$ Furthermore, prescribing NOAC after major bleeding was associated with lower risk of major bleeding recurrence than resuming or not resuming warfarin in patients with $\mathrm{AF}^{48}$ which shows potential prospect for prescribing NOAC after anticoagulation-associated ICH.

The key limitation to this review is that in observational studies the resumption of anticoagulant therapy is probably associated with factors related to future risk such as age, clinical severity or size of the haematoma, and the effects of these on the outcomes of interest cannot be confidently controlled. Data on these factors were not always readily extractable from the included studies, and confounding effects on clinical outcomes after resuming anticoagulant therapy could not be avoided in study-level meta-analysis. In addition, current evidence is based on studies recruiting participants with mixed indication of oral anticoagulants. The publication bias assessment for long-term mortality may be underpowered because of the small number of studies $(<10)$ being included for meta-analysis. The analysis of factors associated with resuming anticoagulant therapy should be interpreted with caution because of multiple binary comparisons with variable heterogeneity. Further studies with rigorous stratification of confounding factors are needed. As these data are based on non-randomised, non-blinded, observational studies of variable quality, we do not have truly reliable evidence on which to base recommendations for management.

In summary, the current evidence from observational studies suggests that resuming anticoagulant therapy after anticoagulation-associated ICH is associated with beneficial effects related to the prevention of thromboembolic events without clear influence on ICH recurrence and survival. Given increased burden of anticoagulation-associated ICH related to anticoagulation for AF and other cardiac conditions in ageing populations, there is an urgent need for RCT-based evidence to guide clinical practice.

\section{Author affiliations}

${ }^{1}$ Department of Radiology, South Campus, Ren Ji Hospital, School of Medicine,

Shanghai Jiao Tong University, Shanghai, China

${ }^{2}$ The George Institute for Global Health, Faculty of Medicine, University of New South Wales, Sydney, New South Wales, Australia

${ }^{3}$ Department of Cardiology, Peking University Third Hospital, Beijing, China 
${ }^{4}$ Department of Neurology, Royal Prince Alfred Hospital, Sydney Health Partners, Sydney, New South Wales, Australia

${ }^{5}$ Sydney Medical School, University of Sydney, Sydney, New South Wales, Australia ${ }^{6}$ Department of Geriatrics, South Campus, Ren Ji Hospital, School of Medicine,

Shanghai Jiao Tong University, Shanghai, China

${ }^{7}$ The Charles Perkins Centre, University of Sydney, Sydney, New South Wales, Australia

${ }^{8}$ Department of Epidemiology and Biostatistics, Imperial College London, London, United Kingdom

${ }^{9}$ The George Institute China, Peking University Health Science Center, Beijing, China

Contributors Conception and design: JS, ZZ, RIL and CSA. Literature search and data extraction: ZZ, JY, JS and MLH. Analysis and interpretation of data: ZZ, CC, $\mathrm{CD}, \mathrm{RIL}, \mathrm{BN}$ and MLH. Initial drafting of manuscript: ZZ. Critical revision of the manuscript for intellectual content: all authors. Final approval of the manuscript: all authors.

Funding ZZ holds a research grant from Shanghai Health and Family Planning Commission (No. 20144Y0119, 2015-2017), an overseas visiting funding from Ren Ji Hospital, School of Medicine, Shanghai Jiao Tong University (2016-2018), and a Scientia PhD Scholarship from the UNSW Sydney (2018-2022). CSA holds a senior principal research fellowship of the National Health and Medical Research Council (NHMRC) of Australia. MLH holds a National Heart Foundation Future Leader Fellowship, level 2 (100034, 2014-2017) and a NHMRC Career Development Fellowship, Level 2, APP1141328.

Disclaimer The work was done by the authors with no involvement of the funder in the design or conduct of the study; collection, management, analysis or interpretation of the data; preparation, review or approval of the manuscript; or decision to submit the manuscript for publication.

Competing interests None declared.

Patient consent Not required.

Provenance and peer review Not commissioned; externally peer reviewed.

Data sharing statement All data are freely available within the appendices.

Open Access This is an Open Access article distributed in accordance with the Creative Commons Attribution Non Commercial (CC BY-NC 4.0) license, which permits others to distribute, remix, adapt, build upon this work non-commercially, and license their derivative works on different terms, provided the original work is properly cited and the use is non-commercial. See: http://creativecommons.org/ licenses/by-nc/4.0/

(c) Article author(s) (or their employer(s) unless otherwise stated in the text of the article) 2018. All rights reserved. No commercial use is permitted unless otherwise expressly granted.

\section{REFERENCES}

1. Coleman $\mathrm{Cl}$, Sobieraj DM, Winkler S, et al. Effect of pharmacological therapies for stroke prevention on major gastrointestinal bleeding in patients with atrial fibrillation. Int J Clin Pract 2012;66:53-63.

2. Schelleman H, Brensinger CM, Bilker WB, et al. Antidepressantwarfarin interaction and associated gastrointestinal bleeding risk in a case-control study. PLoS One 2011;6:e21447.

3. Cannegieter SC, Rosendaal FR, Briët E. Thromboembolic and bleeding complications in patients with mechanical heart valve prostheses. Circulation 1994;89:635-41.

4. Hart RG, Tonarelli SB, Pearce LA. Avoiding central nervous system bleeding during antithrombotic therapy: recent data and ideas. Stroke 2005;36:1588-93.

5. Cervera A, Amaro S, Chamorro A. Oral anticoagulant-associated intracerebral hemorrhage. J Neurol 2012;259:212-24.

6. Wiedermann CJ, Stockner I. Warfarin-induced bleeding complications - clinical presentation and therapeutic options. Thromb Res 2008;122(Suppl. 2):S13-8.

7. Rosand J, Eckman MH, Knudsen KA, et al. The effect of warfarin and intensity of anticoagulation on outcome of intracerebral hemorrhage. Arch Intern Med 2004;164:880-4.

8. Fang MC, Go AS, Chang Y, et al. Thirty-day mortality after ischemic stroke and intracranial hemorrhage in patients with atrial fibrillation on and off anticoagulants. Stroke 2012;43:1795-9.

9. Chai-Adisaksopha C, Hillis C, Monreal M, et al. Thromboembolic events, recurrent bleeding and mortality after resuming anticoagulant following gastrointestinal bleeding. a meta-analysis. Thromb Haemost 2015;114:819-25.

10. Romualdi E, Micieli E, Ageno W, et al. Oral anticoagulant therapy in patients with mechanical heart valve and intracranial haemorrhage. a systematic review. Thromb Haemost 2009;101:290-7.

11. Hawryluk GW, Austin JW, Furlan JC, et al. Management of anticoagulation following central nervous system hemorrhage in patients with high thromboembolic risk. J Thromb Haemost 2010;8:1500-8.

12. Flynn RW, MacDonald TM, Murray GD, et al. Systematic review of observational research studying the long-term use of antithrombotic medicines following intracerebral hemorrhage. Cardiovasc Ther 2010;28:177-84.

13. Moher D, Liberati A, Tetzlaff $\mathrm{J}$, et al. Preferred reporting items for systematic reviews and meta-analyses: the PRISMA Statement. Open Med 2009;3:e123-30.

14. Higgins JP, Altman DG, Gøtzsche PC, et al. The Cochrane Collaboration's tool for assessing risk of bias in randomised trials. BMJ 2011;343:d5928.

15. Wells GA, Shea B, O'Connell D, et al. The Newcastle-Ottawa Scale (NOS) for Assessing the Quality of Nonrandomised Studies in MetaAnalyses. The Ottawa Hospital Research Institute 2015 (accessed 20 Feb 2015).

16. DerSimonian R, Laird N. Meta-analysis in clinical trials. Control Clin Trials 1986;7:177-88.

17. De Vleeschouwer S, Van Calenbergh F, van Loon J, et al. Risk analysis of thrombo-embolic and recurrent bleeding events in the management of intracranial haemorrhage due to oral anticoagulation. Acta Chir Belg 2005;105:268-74.

18. Claassen DO, Kazemi N, Zubkov AY, et al. Restarting anticoagulation therapy after warfarin-associated intracerebral hemorrhage. Arch Neurol 2008;65:1313-8.

19. Majeed A, Kim YK, Roberts RS, et al. Optimal timing of resumption of warfarin after intracranial hemorrhage. Stroke 2010;41:2860-6.

20. Yung D, Kapral MK, Asllani E, et al. Reinitiation of anticoagulation after warfarin-associated intracranial hemorrhage and mortality risk: the Best Practice for Reinitiating Anticoagulation Therapy After Intracranial Bleeding (BRAIN) study. Can J Cardiol 2012;28:33-9.

21. Vidal-Jordana A, Barroeta-Espar I, Sáinz Pelayo MP, et al. [Intracerebral hemorrhage in anticoagulated patients: what do we do afterwards?]. Neurologia 2012;27:136-42.

22. Gathier CS, Algra A, Rinkel GJ, et al. Long-term outcome after anticoagulation-associated intracerebral haemorrhage with or without restarting antithrombotic therapy. Cerebrovasc Dis 2013;36:33-7.

23. Teo KC, Mahboobani NR, Lee R, et al. Warfarin associated intracerebral hemorrhage in Hong Kong Chinese. Neurol Res 2014;36:143-9.

24. Kuramatsu JB, Gerner ST, Schellinger PD, et al. Anticoagulant reversal, blood pressure levels, and anticoagulant resumption in patients with anticoagulation-related intracerebral hemorrhage. JAMA 2015;313:824-36.

25. Nielsen PB, Larsen TB, Skjøth F, et al. Restarting anticoagulant treatment after intracranial hemorrhage in patients with atrial fibrillation and the impact on recurrent stroke, mortality, and bleeding: a nationwide cohort study. Circulation 2015;132:517-25.

26. Osaki M, Koga M, Maeda K, et al. A multicenter, prospective, observational study of warfarin-associated intracerebral hemorrhage: The SAMURAI-WAICH study. J Neurol Sci 2015;359(1-2):72-7.

27. Witt DM, Clark NP, Martinez K, et al. Risk of thromboembolism, recurrent hemorrhage, and death after warfarin therapy interruption for intracranial hemorrhage. Thromb Res 2015;136:1040-4.

28. Mirzayan MJ, Calvelli K, Capelle HH, et al. Subdural hematoma and oral anticoagulation: a therapeutic dilemma from the neurosurgical point of view. J Neurol Surg A Cent Eur Neurosurg 2016;77:31-5.

29. Biffi A, Kuramatsu JB, Leasure $A$, et al. Oral Anticoagulation and Functional Outcome after Intracerebral Hemorrhage. Ann Neurol 2017;82:755-65

30. Murthy SB, Gupta A, Merkler AE, et al. Restarting anticoagulant therapy after intracranial hemorrhage: a systematic review and metaanalysis. Stroke 2017;48:1594-600.

31. Korompoki E, Filippidis FT, Nielsen PB, et al. Long-term antithrombotic treatment in intracranial hemorrhage survivors with atrial fibrillation. Neurology 2017;89:687-96.

32. Chai-Adisaksopha $\mathrm{C}$, lorio $\mathrm{A}$, Hillis $\mathrm{C}$, et al. Warfarin resumption following anticoagulant-associated intracranial hemorrhage: A systematic review and meta-analysis. Thromb Res 2017;160:97-104.

33. Ottosen TP, Grijota M, Hansen ML, et al. Use of antithrombotic therapy and long-term clinical outcome among patients surviving intracerebral Hemorrhage. Stroke 2016;47:1837-43. 
34. US National Library of medicine. Apixaban Versus Antiplatelet Drugs or no Antithrombotic Drugs After Anticoagulation-associated Intracerebral Haemorrhage in Patients With Atrial Fibrillation (APACHE-AF). 2015. https://www.clinicaltrials.gov/ct2/show/ NCT02565693?term=APACHE-AF\&rank=1

35. Eckman MH, Rosand J, Knudsen KA, et al. Can patients be anticoagulated after intracerebral hemorrhage? A decision analysis. Stroke 2003;34:1710-6.

36. Pennlert J, Overholser R, Asplund K, et al. Optimal Timing of Anticoagulant Treatment After Intracerebral Hemorrhage in Patients With Atrial Fibrillation. Stroke 2017;48:314-20.

37. Hemphill JC, Greenberg SM, Anderson CS, et al. Guidelines for the management of spontaneous intracerebral hemorrhage: a guideline for healthcare professionals from the American Heart Association/ American Stroke Association. Stroke 2015;46:2032-60.

38. AlKherayf $\mathrm{F}, \mathrm{Xu} \mathrm{Y}$, Westwick $\mathrm{H}$, et al. Timing of anticoagulant reinitiation following intracerebral hemorrhage in mechanical heart valves: Survey of neurosurgeons and thrombosis experts. Clin Neurol Neurosurg 2017;154:23-7.

39. AlKherayf F, Xu Y, Gandara E, et al. Timing of vitamin K antagonist re-initiation following intracranial hemorrhage in mechanical heart valves: Systematic review and meta-analysis. Thromb Res 2016;144:152-7.

40. BioMed Central Ltd. REstart or STop Antithrombotics Randomised Trial. 2018. http://www.isrctn.com/ISRCTN71907627

41. US National Library of medicine. REstart or STop Antithrombotic Randomised Trial in France (RESTART-Fr). 2017. https://www. clinicaltrials.gov/ct2/show/NCT02966119?term=RESTART\&rank=1
42. Ruff CT, Giugliano RP, Braunwald E, et al. Comparison of the efficacy and safety of new oral anticoagulants with warfarin in patients with atrial fibrillation: a meta-analysis of randomised trials. Lancet 2014;383:955-62.

43. Chatterjee S, Sardar P, Biondi-Zoccai G, et al. New oral anticoagulants and the risk of intracranial hemorrhage: traditional and Bayesian meta-analysis and mixed treatment comparison of randomized trials of new oral anticoagulants in atrial fibrillation. JAMA Neurol 2013;70:1486-90.

44. Van de Werf F, Brueckmann M, Connolly SJ, et al. A comparison of dabigatran etexilate with warfarin in patients with mechanical heart valves: the randomized, phase II study to evaluate the safety and pharmacokinetics of oral dabigatran etexilate in patients after heart valve replacement (RE-ALIGN). Am Heart J 2012;163:931-7.

45. Eikelboom JW, Connolly SJ, Brueckmann M, et al. Dabigatran versus warfarin in patients with mechanical heart valves. $N$ Engl J Med 2013;369:1206-14.

46. Hagii J, Tomita H, Metoki N, et al. Characteristics of intracerebral hemorrhage during rivaroxaban treatment: comparison with those during warfarin. Stroke 2014;45:2805-7.

47. Wilson D, Charidimou A, Shakeshaft C, et al. Volume and functional outcome of intracerebral hemorrhage according to oral anticoagulant type. Neurology 2016;86:360-6.

48. Hernandez I, Zhang Y, Brooks MM, et al. Anticoagulation Use and clinical outcomes after major bleeding on dabigatran or warfarin in atrial fibrillation. Stroke 2017;48:159-66. 